\title{
The application of complementary ablation in patients with AVRT
}

\author{
GuangZe $\mathrm{Xu}^{1}$, Zhikui Chen ${ }^{1}$, and Haiyan $\operatorname{Lin}^{1}$ \\ ${ }^{1}$ Li Huili Hospital
}

April 28, 2020

\begin{abstract}
Background:Radiofrequency catheter ablation(RFCA) has now become the standard and effective therapy for paroxysmal supraventricular tachycardias, but the success rates of ablation were reported different in a large number of single-centers. It is estimated that there are still thousands of cases of relapse in China every year, among which the main cases are of accessory pathways(Aps) mediated tachycardia.Objective: The purpose of this study was to compare the efficacy and safety of two different radiofrequency ablation strategies, improve ablation method and reduce the recurrence rate of ablation of accessory pathway mediated tachycardia.Method and Result: 1712 consecutive cases of AVRT with 1741 accessory pathways were analyzed in our retrospectively observational study. They were divided into group S and group M (undergone complementary ablation) according to different strategies of RFCA. 882 cases with 898 Aps were enrolled in group S versus 830 cases with 843 Aps in group $\mathrm{M}$. The results showed the cumulative recurrence number (rate) at the 1st, 3rd, 6th, 12th, and 24th month after ablation were $4(0.5 \%)$ vs $17(1.9 \%) \mathrm{p}=0.007,5(0.6 \%)$ vs $27(3.0 \%) \mathrm{p}=0.000,6(0.7 \%)$ vs $34(3.8 \%) \mathrm{p}=0.000,6(0.7 \%)$ vs $58(6.5 \%) \mathrm{p}=0.000$, $7(0.8 \%)$ vs $63(7.1 \%) \mathrm{p}=0.000$, respectively; Complications of chest pain, over vasovagal reaction, steam pop, angina pectoris, and embolism were rare in both groups; No Valve damage, myocardial infarction, cardiac tamponade, periprocedural death or other serious adverse events occurred in the both groups. Conclusions:The strategy of complementary ablation significantly reduced the recurrence rate of radiofrequency ablation of accessory pathway without increasing the risk of complications.
\end{abstract}

\section{Hosted file}

manuscript.doc available at https ://authorea.com/users/316179/articles/446366-the-applicationof-complementary-ablation-in-patients-with-avrt 